\title{
Static anti-windup synthesis for linear systems with time-varying input delays.
}

\author{
J. V. Flores * J. M. Gomes da Silva Jr. ${ }^{*}$ A. Seuret ${ }^{* *}$ \\ * UFRGS - Department of Electrical Engineering, \\ Av. Osvaldo Aranha 103, 90035-190 Porto Alegre-RS, Brazil. \\ E-mail: jeferson.flores@ufrgs.br,jmgomes@ece.ufrgs.br. \\ ** Department of Automatic Control, GIPSA-Lab CNRS UMR 5216, \\ INRIA NECS project Team \\ Grenoble, France \\ E-mail: alexandre.seuret@gipsa-lab.inpg.fr.
}

\begin{abstract}
This article considers the design of an anti-windup compensator for linear systems subject to time-varying input delays and saturating actuators. Local and global stabilization conditions ensuring both external as well as internal stability of the closed-loop system are derived directly as linear matrix inequalities (LMIs). To compute the anti-windup gains, these conditions are cast into the following optimization problems: maximization of the set of admissible initial conditions, maximization of the bound of admissible $\mathcal{L}_{2}$ disturbances or the maximization of the $\mathcal{L}_{2}$-gain from the disturbance to the regulated output. Simulation examples are provided to illustrate the proposed solution.
\end{abstract}

\section{INTRODUCTION}

Time-delay is a common phenomena in many physical systems such as aircraft stabilization, chemical reactors, communication networks and others. Due to the fact that the presence of delays in control systems can lead to unsatisfactory time-domain performances or even to instability, in the last years great attention has been paid to stability analysis and control of these systems (see Richard [2003], and references). Stability conditions for time-delay systems can be classified into delay-dependent and delay-independent conditions. Concerning the delay independent results, the stability is ensured no matter the size of the delay (Shaked et al. [1998]). On the other hand, in the delay dependent results, the size of the delay is directly taken into account and this fact can lead, especially when the time-delays are small, to less conservative results (Fridman and Shaked [2003]).

Most of the works dealing with time-delays consider invariant delays. However, there is an increasing number of practical systems presenting uncertain and/or timevarying delays in the state and/or input variable. This is a common problem in communications systems where the presence of transmission delay variations is usually unavoidable (Park et al. [2003]). Many approaches have been developed regarding the stability problem for systems with time-varying input delays: Niculescu et al. [1997], Zhang et al. [2005], Mahmoud [2009] and references therein. In Niculescu et al. [1997], the delay was assumed to be a continuous bounded time-varying function and the analysis was presented considering a Razumikhin based framework and a frequency-filtering method. In Zhang et al. [2005] a state/input-delay-dependent stabilization

\footnotetext{
* The first two authors are supported in part by CNPq, Brazil. The third author is partially supported by European Comission through the FeedNetBack project.
}

condition was obtained considering a new integral inequality for quadratic terms and a Lyapunov-Krasovskii approach. Following the same ideas, in Mahmoud [2009] a $\mathcal{L}_{2}$-performance analysis is used to derive the solution for both nominal and polytopic uncertain models.

On the other hand, saturation is an ubiquitous problem in control systems, arising from the fact that actuators cannot delivery signals with unlimited amplitude or energy to the controlled plants. Neglecting actuator limitations can therefore be source of undesirable or even catastrophic behaviors for the closed-loop system (as the lost of the closed-loop stability). One of the most usual ways to mitigate the undesirable effects of the saturation is the anti-windup technique. This technique assumes that a controller was previously designed in order to guarantee some performance requirements and then this controller is modified in order to recover, as much as possible, the performance when saturation occurs (see, for example, the survey Tarbouriech and Turner [2009]).

In the anti-windup context for time-delay systems, we can cite Park et al. [2000], Tarbouriech et al. [2003], Tarbouriech et al. [2004], Zaccarian et al. [2005] and Gomes da Silva Jr. et al. [2006]. In Park et al. [2000], a dynamic anti-windup strategy is considered for systems with input and output delays. In Tarbouriech et al. [2003], the design of a static anti-windup compensator was studied with the aim of enlarging the region of attraction of the closed-loop system. In that work, a method for computing anti-windup gains for systems presenting only input delays is proposed leading to bilinear matrix inequalities (BMIs) conditions. In contrast to Park et al. [2000] and Zaccarian et al. [2005], the proposed technique can be applied to both stable and unstable open-loop systems. In Tarbouriech et al. [2004] and Gomes da Silva Jr. et al. [2006], based on a Lyapunov-Krasosvskii functional and on the application 
of a modified sector condition, global and local delaydependent stabilization conditions are derived. However, it should be pointed out that these works focus, respectively, on systems presenting output or state delays. Furthermore, in the above works the delays are supposed to be fixed.

In this work, the problem of anti-windup design for linear systems with time-varying input delay and actuator saturation is addressed. An anti-windup strategy considering two static compensators is considered: one responsible to correct the actual difference between the computed control signal and the control delivered to the plant and another responsible to correct the delayed difference between then, if the delay value is known at each time. Following the descriptor like approach considered in Gomes da Silva Jr. et al. [2010] and the modified sector condition from Tarbouriech et al. [2004] to take into account the saturation effects, global and local stabilization conditions directly in the LMI form are derived to ensure both $\mathcal{L}_{2}$ input to state stability and the internal stability of the closed-loop system. Provided that the maximal value of the time-varying delay and an upper bound for its derivative are known, convex optimization problems to compute the anti-windup gains are proposed. Simulation examples are presented to illustrate the the proposed solution.

Notation: The $i$ th component of a vector $x$ is denoted by $x_{(i)} \cdot A_{(i)}$ denotes the $i$ th row of a matrix $A \in \mathbb{R}^{n \times n}$ and $A^{T}$ denotes its transpose. $\operatorname{diag}\{x\}$ denotes a diagonal matrix obtained from vector $x, I_{m}$ denotes the $m$-order identity matrix and $0_{m \times n}$ denotes the $m \times n$ null matrix. $\bar{\lambda}(P)$ and $\underline{\lambda}(P)$ denote the maximal and the minimal eigenvalues of $\overline{\mathrm{P}}$, respectively. $\mathcal{C}_{h}=C\left([-h, 0], \mathbb{R}^{n}\right)$ is the Banach space of continuous vector functions mapping the interval $[-h, 0]$ into $\mathbb{R}^{n}$ with the norm $\|\phi\|_{c}=\sup _{-h<t<0}\|\phi(t)\| . \mathcal{C}_{h}^{\nu}$ is the set defined by $\mathcal{C}_{h}^{\nu}=\left\{\phi \in \mathcal{C}_{h} ;\|\phi\|_{c} \leq \nu, \nu>0\right\}$. * denotes symmetric block elements in a matrix.

\section{PROBLEM STATEMENT}

Consider the following linear time-delay system:

$$
\begin{aligned}
& \dot{x}(t)=A x(t)+B u(t-\tau(t))+B_{w} w(t) \\
& y(t)=C_{y} x(t) \\
& z(t)=C_{z} x(t)+D_{z} u(t-\tau(t))
\end{aligned}
$$

with initial conditions $x\left(t_{0}+\theta\right)=\phi(\theta), \forall \theta \in[-h, 0], t_{0} \in$ $\mathbb{R}_{+}, \phi(\theta) \in \mathcal{C}_{h}^{\nu}$. In this case, $x(t) \in \mathbb{R}^{n}$ is the state vector, $u(t) \in \mathbb{R}^{m}$ is the control input, $w(t) \in \mathbb{R}^{l}$ is the external disturbance vector, $y(t) \in \mathbb{R}^{p}$ is the measured output, $z(t) \in \mathbb{R}^{p}$ is the regulated output and $\tau(t)$ corresponds to a time-varying delay that satisfies $0 \leq \tau(t) \leq h, \quad \dot{\tau}(t) \leq$ $d<1$

Matrices $A, B, B_{w}, C_{y}, C_{z}$ and $D_{z}$ are constant real matrices of appropriate dimensions, pairs $(A, B)$ and $\left(C_{y}, A\right)$ are assumed to be stabilizable and detectable, respectively, and we suppose that the control input $u$ is subject to amplitude limitations such that $\left|u_{(i)}(t)\right| \leq u_{0(i)}, \quad u_{0(i)}>$ $0, \quad i=1, \cdots, m$.

The disturbance vector $w(t)$ is assumed to be limited in energy, i.e. $w(t) \in \mathcal{L}_{2}$, and for some scalar $\delta, 0 \leq \frac{1}{\delta}<\infty$, the disturbance $w(t)$ is bounded as follows:

$$
\|w(t)\|_{2}^{2}=\int_{0}^{\infty} w^{T}(t) w(t) d t \leq \frac{1}{\delta}
$$

Considering system (1), we assume therefore that an $n_{c^{-}}$ order dynamic output stabilizing compensator

$$
\begin{aligned}
& \dot{x}_{c}(t)=A_{c} x_{c}(t)+B_{c} u_{c}(t) \\
& y_{c}(t)=C_{c} x_{c}(t)+D_{c} u_{c}(t),
\end{aligned}
$$

where $x_{c}(t) \in \mathbb{R}^{n_{c}}$ is the controller state, $u_{c}(t)=y(t) \in \mathbb{R}^{p}$ is the controller input and $y_{c}(t) \in \mathbb{R}^{m}$ is the controller output has been designed in order to guarantee some performance requirements and the stability of the closedloop system in the absence of the control saturation. Matrices $A_{c}, B_{c}, C_{c}$ and $D_{c}$ are of appropriate dimensions. In consequence of the control bounds, the control signal to be injected in the system is a saturated one, that is,

$$
u(t)=\operatorname{sat}\left(y_{c}(t)\right)=\operatorname{sat}\left(C_{c} x_{c}(t)+D_{c} y(t)\right)
$$

with each component of $\operatorname{sat}\left(y_{c}(t)\right)$ classically defined as $\operatorname{sat}\left(y_{c(i)}(t)\right) \triangleq \operatorname{sign}\left(y_{c(i)}(t)\right) \min \left(\left|y_{c(i)}(t)\right|, u_{0(i)}\right), \quad i=$ $1, \ldots, m$.

In order to mitigate the undesirable effects of windup caused by input saturation, two static anti-windup compensators $E_{c}\left(\operatorname{sat}\left(y_{c}(t)\right)-y_{c}(t)\right), \quad E_{c} \in \mathbb{R}^{n_{c} \times m}$, and $F_{c}\left(\operatorname{sat}\left(y_{c}(t-\tau(t))\right)-y_{c}(t-\tau(t))\right), F_{c} \in \mathbb{R}^{n_{c} \times m}$, can be added to the controller. Thus, replacing the control signal in (1) by its saturated version (4), considering the dynamic controller and the proposed anti-windup strategy, it follows that the closed loop system reads:

$$
\begin{aligned}
\dot{x}(t)= & A x(t)+B \operatorname{sat}\left(y_{c}(t-\tau(t))\right)+B_{w} w(t) \\
y(t)= & C_{y} x(t) \\
z(t)= & C_{z} x(t)+D_{z} \operatorname{sat}\left(y_{c}(t-\tau(t))\right) \\
\dot{x}_{c}(t)= & A_{c} x_{c}(t)+B_{c} y(t)-E_{c} \Psi(u(t)) \\
& -F_{c} \Psi(u(t-\tau(t))) \\
y_{c}(t)= & C_{c} x_{c}(t)+D_{c} y(t),
\end{aligned}
$$

with $\Psi(u(t))=u(t)-\operatorname{sat}(u(t))$ being a decentralized deadzone nonlinearity.

Given $h$ and $d$, we are interested in the synthesis of the anti-windup compensator gains $E_{c}$ and $F_{c}$ in order to address the following problems:

- $\mathcal{L}_{2}$-stabilization: all trajectories of the closed-loop system (5) remain bounded for any disturbance satisfying (2) for a certain $\delta$, i.e. the input-to-state stability should be ensured. Moreover, the controller shall ensure an upper bound for the $\mathcal{L}_{2}$-gain between the disturbance $w(t)$ and the regulated output $z(t)$, which corresponds to the disturbance rejection problem.

- Internal Stabilization: in the absence of disturbances, the internal asymptotic stability of the closed-loop system (5) shall be guaranteed for all initial conditions belonging to a certain set $\mathcal{D}_{0}$ of functions $\phi \in \mathcal{C}_{h}^{\nu}$, i.e. the set $\mathcal{D}_{0}$ is included in the basin of attraction of the origin of the closed-loop system (5).

\section{PRELIMINARIES}

Define the augmented state $\xi(t)=\left[x(t)^{T} \quad x_{c}(t)^{T}\right]^{T} \quad \in$ $\mathbb{R}^{n+n_{c}}$ an the following matrices: $\mathbb{A}=\left[\begin{array}{cc}A & 0 \\ B_{c} C_{y} & A_{c}\end{array}\right], \mathbb{A}_{d}=\left[\begin{array}{cc}B D_{c} C_{y} & B C_{c} \\ 0 & 0\end{array}\right], \mathbb{B}_{w}=\left[\begin{array}{c}B_{w} \\ 0\end{array}\right]$,
$\mathbb{B}_{1}=\left[\begin{array}{c}B \\ 0\end{array}\right], \mathbb{B}_{2}=\left[\begin{array}{c}0 \\ I_{n_{c}}\end{array}\right], \mathbb{B}_{E}=\mathbb{B}_{2} E_{c}, \mathbb{B}_{F}=\mathbb{B}_{1}+\mathbb{B}_{2} F_{c}$,
$\mathbb{C}_{z}=\left[\begin{array}{ll}C_{z} & 0\end{array}\right], \mathbb{C}_{d z}=\left[\begin{array}{ll}D_{z} D_{c} C_{y} & D_{z} C_{c}\end{array}\right], \mathbb{D}_{z}=D_{z}$ and
$\mathbb{K}=\left[\begin{array}{ll}D_{c} C_{y} & C_{c}\end{array}\right]$. 
In this case, it is possible to represent the closed loop system (5) as follows:

$$
\begin{aligned}
\dot{\xi}(t)= & \mathbb{A} \xi(t)+\mathbb{A}_{d} \xi(t-\tau(t))-\mathbb{B}_{E} \Psi(\mathbb{K} \xi(t)) \\
& -\mathbb{B}_{F} \Psi(\mathbb{K} \xi(t-\tau(t)))+\mathbb{B}_{w} w(t), \\
z(t)= & \mathbb{C}_{z} \xi(t)+\mathbb{C}_{d z} \xi(t-\tau(t))-\mathbb{D}_{z} \Psi(\mathbb{K} \xi(t-\tau(t)))
\end{aligned}
$$

with initial conditions $\phi_{\xi}$ defined on $[-h, 0]$, i.e. $\phi_{\xi}=$ $\xi(\theta), \theta \in[-h, 0]$.

Remark 1. Based on (6), it is possible to consider systems with both input and state delays, provided that the timevarying delay is exactly the same at each instant. In this case, the open-loop system (1) reads

$$
\begin{aligned}
& \dot{x}(t)=A x(t)+A_{d} x(t-\tau(t))+B u(t-\tau(t))+B_{w} w(t) \\
& y(t)=C_{y} x(t) \\
& z(t)=C_{z} x(t)+C_{d z} x(t-\tau(t))+D_{z} u(t-\tau(t))
\end{aligned}
$$

and the augmented matrices $\mathbb{A}_{d}$ and $\mathbb{C}_{d z}$ in (6) must be replaced by $\mathbb{A}_{d}=\left[\begin{array}{cc}A_{d}+B D_{c} C_{y} & B C_{c} \\ 0 & 0\end{array}\right]$ and $\mathbb{C}_{d z}=$ $\left[C_{d z}+D_{z} D_{c} C_{y} D_{z} C_{c}\right]$.

Considering a matrix $\mathbb{G}_{0} \in \mathbb{R}^{m \times\left(n+n_{c}\right)}$ and defining the polyhedral set

$$
\mathcal{S}_{0} \triangleq\left\{\xi \in \mathbb{R}^{n+n_{c}} ;\left|\left(\mathbb{K}_{(i)}-\mathbb{G}_{0(i)}\right) \xi\right| \leq u_{0(i)}, i=1, \ldots, m\right\}
$$

the following Lemma, concerning $\Psi(\mathbb{K} \xi)$ can be stated.

Lemma 2. (Tarbouriech et al. [2004]) Consider the deadzone nonlinearity $\Psi(\mathbb{K} \xi)$. If $\xi \in \mathcal{S}_{0}$ then the relation

$$
\Psi(\mathbb{K} \xi)^{T} T_{0}\left[\Psi(\mathbb{K} \xi)-\mathbb{G}_{0} \xi\right] \leq 0
$$

is verified for any diagonal positive definite matrix $T_{0} \in$ $\Re^{m \times m}$.

\section{MAIN RESULT}

The following theorem considers the anti-windup synthesis in a local context. In this case, the stability is ensured provided that the initial conditions of the closed loop system and the disturbances belong to certain admissible sets.

Theorem 3. If there exist symmetric positive definite matrices $Q_{1}, X, U \in \mathbb{R}^{\left(n+n_{c}\right) \times\left(n+n_{c}\right)}$, positive definite diagonal matrices $S_{0}, \quad S_{1} \in \mathbb{R}^{m \times m}$, matrices $Q_{2}, Q_{3} \in$ $\mathbb{R}^{\left(n+n_{c}\right) \times\left(n+n_{c}\right)}, W_{0}, W_{1} \in \mathbb{R}^{m \times\left(n+n_{c}\right)}, Y_{E}, Y_{F} \in \mathbb{R}^{n_{c} \times m}$ and positive scalars $\gamma$ and $\mu$ satisfying the matrix inequalities

$$
\left[\begin{array}{ccccccc}
\Sigma_{1} & \Sigma_{2} & \Sigma_{3} & \Sigma_{4} & \Sigma_{5} & \Sigma_{6} & \Sigma_{7} \\
* & \Sigma_{8} & 0 & W_{1}^{T} & 0 & 0 & Q_{1} \mathbb{C}_{d z}^{T} \\
* & * & -2 S_{0} & 0 & 0 & 0 & 0 \\
* & * & * & -2 S_{1} & 0 & 0 & -S_{1} \mathbb{D}_{z}^{T} \\
* & * & * & * & -2 h Q_{1}+h U & 0 & 0 \\
* & * & * & * & * & -I & 0 \\
* & * & * & * & * & * & -\gamma I
\end{array}\right]<0
$$

$$
\begin{gathered}
{\left[\begin{array}{cc}
Q_{1} & Q_{1} \mathbb{K}_{(i)}^{T}-W_{0(i)}^{T} \\
* & \mu u_{0(i)}^{2}
\end{array}\right] \geq 0, i=1, \cdots, m} \\
{\left[\begin{array}{cc}
Q_{1} & Q_{1} \mathbb{K}_{(i)}^{T}-W_{1(i)}^{T} \\
* & \mu u_{0(i)}^{2}
\end{array}\right] \geq 0, i=1, \cdots, m} \\
\mu-\delta \leq 0
\end{gathered}
$$

where $\Sigma_{1}=\left[\begin{array}{cc}Q_{2}+Q_{2}^{T}+X-U / h & Q_{1} \mathbb{A}^{T}-Q_{2}^{T}+Q_{3} \\ * & -Q_{3}-Q_{3}^{T}\end{array}\right]$, $\Sigma_{2}=\left[\begin{array}{c}U / h \\ \mathbb{A}_{d} Q_{1}\end{array}\right], \Sigma_{3}=\left[\begin{array}{c}W_{0}^{T} \\ -\mathbb{B}_{2} Y_{E}\end{array}\right], \Sigma_{4}=\left[\begin{array}{c}-Q_{3}-Q_{3}^{T} \\ 0 \\ -\mathbb{B}_{1} S_{1}-\mathbb{B}_{2} Y_{F}\end{array}\right]$, $\Sigma_{5}=h\left[\begin{array}{l}Q_{2}^{T} \\ Q_{3}^{T}\end{array}\right], \Sigma_{6}=\left[\begin{array}{c}0 \\ \mathbb{B}_{w}\end{array}\right], \Sigma_{7}=\left[\begin{array}{c}Q_{1} \mathbb{C}_{z}^{T} \\ 0\end{array}\right]$ and $\Sigma_{8}=$ $(d-1) X-U / h$, then the anti-windup gains $E_{c}=Y_{E} S_{0}^{-1}$ and $F_{c}=Y_{F} S_{1}^{-1}$ ensure that:

(1) the trajectories of system (6) are bounded for every initial condition satisfying

$$
\begin{aligned}
\beta= & {\left[\bar{\lambda}\left(Q_{1}^{-1}\right)+h \bar{\lambda}\left(Q_{1}^{-1} X Q_{1}^{-1}\right)\right]\left\|\phi_{\xi}\right\|_{c}^{2} } \\
& +\frac{h^{2}}{2} \bar{\lambda}\left(Q_{1}^{-1} U Q_{1}^{-1}\right)\left\|\dot{\phi}_{\xi}\right\|_{c}^{2} \leq \mu^{-1}-\delta^{-1}
\end{aligned}
$$

(2) $\|z\|_{2}^{2} \leq \gamma\|w\|_{2}^{2}+\gamma V(0)$

(3) when $w=0$, the closed-loop system origin is locally asymptotically stable and, for all initial conditions satisfying $\beta \leq \mu^{-1}$, the corresponding trajectories converge asymptotically to the origin.

Proof. Consider the following Lyapunov-Krasovskii functional, proposed in Fridman and Shaked [2002] for dealing with time-varying delays:

$$
\begin{aligned}
V(t)= & \xi^{T}(t) P_{1} \xi(t)+\int_{t-\tau(t)}^{t} \xi^{T}(s) M \xi(s) d s \\
& +\int_{-h}^{0} \int_{t+\theta}^{t} \dot{\xi}^{T}(s) R \dot{\xi}(s) d s d \theta
\end{aligned}
$$

with $P_{1}, M$ and $R$ being symmetric positive define matrices. From the definition of $V(t)$, it follows that:

$$
\begin{aligned}
V(0) \leq & \xi^{T}(0) P_{1} \xi(0)+\int_{-h}^{0} \xi^{T}(s) M \xi(s) d s \\
& +\int_{-h}^{0} \int_{\theta}^{0} \dot{\xi}^{T}(s) R \dot{\xi}(s) d s d \theta \\
\leq & {\left[\bar{\lambda}\left(P_{1}\right)+h \bar{\lambda}(M)\right]\left\|\phi_{\xi}\right\|_{c}^{2}+\frac{h^{2}}{2} \bar{\lambda}(R)\left\|\dot{\phi}_{\xi}\right\|_{c}^{2} }
\end{aligned}
$$

Define now $J(t)=\dot{V}(t)-w^{T}(t) w(t)+\frac{1}{\gamma} z^{T}(t) z(t)$. If $J(t)<0$, it follows that

$$
\begin{aligned}
\int_{0}^{T} J(t) d t= & V(T)-V(0)-\int_{0}^{T} w^{T}(t) w(t) d t \\
& +\frac{1}{\gamma} \int_{0}^{T} z^{T}(t) z(t) d t<0
\end{aligned}
$$

which implies that

$$
V(T) \leq V(0)+\|w(t)\|_{2}^{2} \leq \beta+\delta^{-1} \leq \mu^{-1}
$$

$\forall w(t)$ satisfying $(2)$ and $\forall \phi_{\xi}$ such that $\beta+\delta^{-1} \leq \mu^{-1}$. Hence, one gets $\xi(T)^{T} P_{1} \xi(T) \leq V(T) \leq \mu^{-1}$, i.e. for all $T>0$ the trajectories of the system do not leave the set $\mathcal{E}\left(P_{1}, \mu^{-1}\right)=\left\{\xi \in \mathbb{R}^{n+n c} ; \xi(t)^{T} P_{1} \xi(t) \leq \mu^{-1}\right\}$. Moreover, for $T \rightarrow \infty$, (16) yields $\|z\|_{2}^{2} \leq \gamma\|w\|_{2}^{2}+\gamma V(0)$.

In the sequel, we show that (10)-(13) imply that $J(t)<0$, provided that $\phi_{\xi}$ is such that $\beta+\delta^{-1} \leq \mu^{-1}$ and $w(t)$ satisfies (2).

Noting that $\mathbb{A} \xi(t)-\dot{\xi}(t)+\mathbb{A}_{d} \xi(t-\tau(t))-\mathbb{B}_{E} \Psi(\mathbb{K} \xi(t))-$ $\mathbb{B}_{F} \Psi(\mathbb{K} \xi(t-\tau(t)))+\mathbb{B}_{w} w(t)=0$, it follows that the derivative of the Lyapunov-Krasovskii functional $V(t)$ is given by: 


$$
\begin{aligned}
\dot{V}(t)= & \xi^{T}(t) P_{1} \dot{\xi}(t)+\dot{\xi}^{T}(t) P_{1} \xi(t)+\xi^{T}(t) M \xi(t) \\
& -(1-\dot{\tau}(t)) \xi^{T}(t-\tau(t)) M \xi(t-\tau(t)) \\
& +h \dot{\xi}(t)^{T} R \dot{\xi}(t)-\int_{-h}^{0} \dot{\xi}^{T}(t+\theta) R \dot{\xi}(t+\theta) d \theta \\
& +2 \xi^{T}(t) P_{2}^{T}\left[\mathbb{A} \xi(t)-\dot{\xi}(t)+\mathbb{A}_{d} \xi(t-\tau(t))\right. \\
& \left.-\mathbb{B}_{E} \Psi(\mathbb{K} \xi(t))-\mathbb{B}_{F} \Psi(\mathbb{K} \xi(t-\tau(t)))+\mathbb{B}_{w} w(t)\right] \\
& +2 \dot{\xi}^{T}(t) P_{3}^{T}\left[\mathbb{A} \xi(t)-\dot{\xi}(t)+\mathbb{A}_{d} \xi(t-\tau(t))\right. \\
& -\mathbb{B}_{E} \Psi(\mathbb{K} \xi(t))-\mathbb{B}_{F} \Psi\left(\mathbb{K} \xi(t-\tau(t))+\mathbb{B}_{w} w(t)\right] .
\end{aligned}
$$

Introducing the vector $\bar{\xi}(t)=\left[\begin{array}{ll}\xi^{T}(t) & \dot{\xi}^{T}(t)\end{array}\right]^{T}$ and the matrix $P=\left[\begin{array}{rr}P_{1} & 0 \\ P_{2} & P_{3}\end{array}\right]$, we will follow the descriptor approach considered in Gomes da Silva Jr. et al. [2010]. In this case, $J(t)$ can be expressed as:

$$
\begin{aligned}
J(t)= & \bar{\xi}^{T}(t) \mathcal{L} \bar{\xi}(t)+2 \bar{\xi}^{T}(t) P^{T}\left[0 \mathbb{A}_{d}^{T}\right]^{T} \xi(t-\tau(t)) \\
& -2 \bar{\xi}^{T}(t) P^{T}\left[\begin{array}{ll}
0 & \mathbb{B}_{E}^{T}
\end{array}\right]^{T} \Psi(\mathbb{K} \xi(t)) \\
& -2 \bar{\xi}^{T}(t) P^{T}\left[\begin{array}{ll}
0 & \mathbb{B}_{F}^{T}
\end{array}\right]^{T} \Psi(\mathbb{K} \xi(t-\tau(t))) \\
& +2 \bar{\xi}^{T}(t) P^{T}\left[0 \mathbb{B}_{w}^{T}\right]^{T} w(t)+\xi^{T}(t) M \xi(t) \\
& -\xi^{T}(t-\tau(t))(1-\dot{\tau}(t)) M \xi(t-\tau(t)) \\
& +h \dot{\xi}^{T}(t) R \dot{\xi}(t)-\int_{t-h}^{t} \dot{\xi}^{T}(s) R \dot{\xi}(s) d s \\
& -w^{T}(t) w(t)+\frac{1}{\gamma} z^{T}(t) z(t)
\end{aligned}
$$

with $\mathcal{L}=\left[\begin{array}{cc}0 & I \\ \mathbb{A} & -I\end{array}\right]^{\prime} P+P^{\prime}\left[\begin{array}{cc}0 & I \\ \mathbb{A} & -I\end{array}\right]$.

Consider the sets $\mathcal{S}_{0}$ defined in (8) and $\mathcal{S}_{1} \triangleq\{\xi \in$ $\left.\mathbb{R}^{n+n_{c}} ;\left|\left(\mathbb{K}_{(i)}-\mathbb{G}_{1(i)}\right) \xi\right| \leq u_{0(i)}, i=1, \ldots, m\right\}$. Provided that $\xi(t) \in \mathcal{S}_{0}$ and $\xi(t-\tau(t)) \in \mathcal{S}_{1}$ then, from Lemma 2 , it follows that:

$$
\begin{aligned}
J(t) \leq & J(t)-2 \Psi(\mathbb{K} \xi(t))^{T} T_{0}\left[\Psi(\mathbb{K} \xi(t))-\mathbb{G}_{0} \xi(t)\right] \\
& -2 \Psi(\mathbb{K} \xi(t-\tau(t)))^{T} T_{1}[\Psi(\mathbb{K} \xi(t-\tau(t))) \\
& \left.-\mathbb{G}_{1} \xi(t-\tau(t))\right]
\end{aligned}
$$

where $T_{0}$ and $T_{1}$ are diagonal positive definite matrices.

Applying now the Jensen's inequality (see Gu et al. [2003] p.322), we have $-\int_{t-h}^{t} \dot{\xi}^{T}(s) R \dot{\xi}(s) d s \leq-(\xi(t)-\xi(t-$ $\tau(t))^{T} \frac{R}{h}(\xi(t)-\xi(t-\tau(t))$. Hence, it follows that $J(t) \leq$ $\eta^{T}(t) \Gamma \eta(t)+\frac{1}{\gamma} z^{T}(t) z(t)$, with $\eta(t)=\left[\bar{\xi}^{T}(t) \quad \xi^{T}(t-\right.$ $\left.\tau(t)) \quad \Psi^{T}(\mathbb{K} \xi(t)) \Psi^{T}(\mathbb{K} \xi(t-\tau(t))) \quad w^{T}(t)\right]^{T}$ and

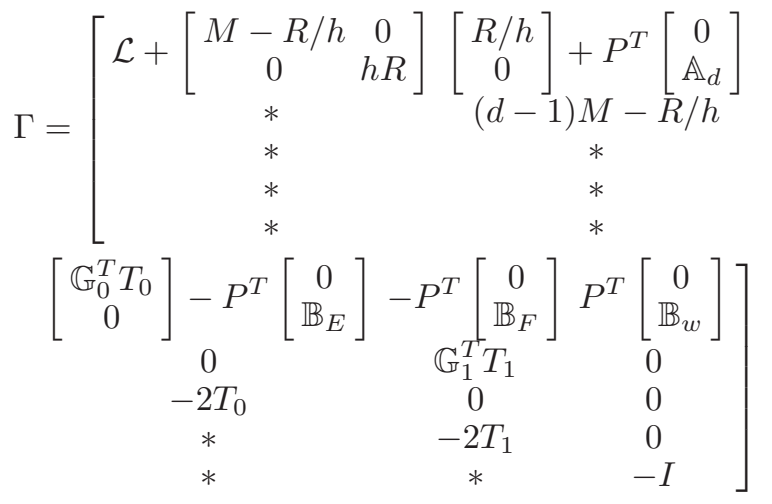

Suppose now that $\Gamma<0$. Applying the Schur's complement to the terms $\left[\begin{array}{cc}0 & 0 \\ 0 & h R\end{array}\right]$ and $\frac{1}{\gamma} z^{T}(t) z(t)$, it follows that

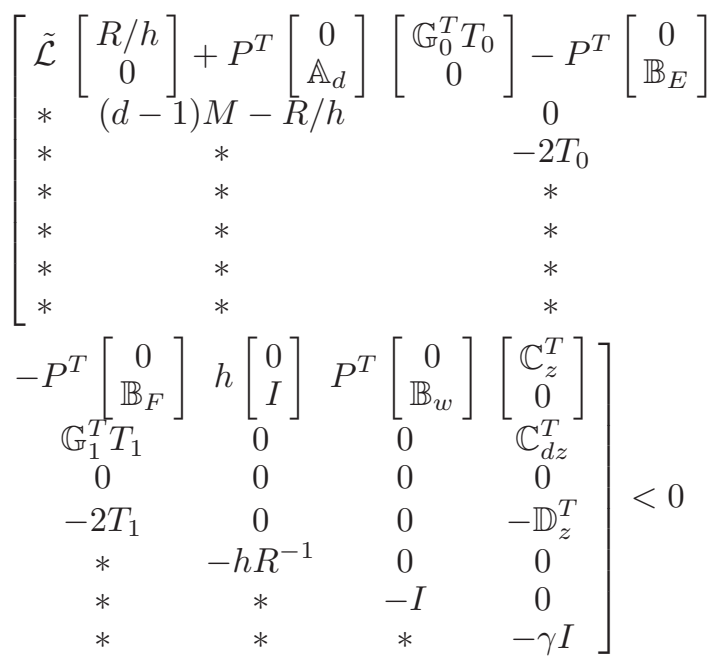

with $\tilde{\mathcal{L}}=\mathcal{L}+\left[\begin{array}{cc}M-R / h & 0 \\ 0 & 0\end{array}\right]$ is verified. Hence, the satisfaction of $(20)$ results in $J(t)<0$. Note also that if (20) is satisfied, one has $\tilde{\mathcal{L}}<0$, which implies that $-P_{3}^{T}-P_{3}$ is negative definite. Hence, since $P_{1}>0$, it follows that matrix $P$ is invertible. Denote now the matrix $P^{-1}=Q=\left[\begin{array}{cc}Q_{1} & 0 \\ Q_{2} & Q_{3}\end{array}\right]$ and define a block diagonal matrix $\Xi=\operatorname{diag}\left\{Q, Q_{1}, T_{0}^{-1}, T_{1}^{-1}, I, I, I\right\}$. By pre and postmultiplying (20) by $\Xi^{T}$ and $\Xi$ respectively, one obtains:

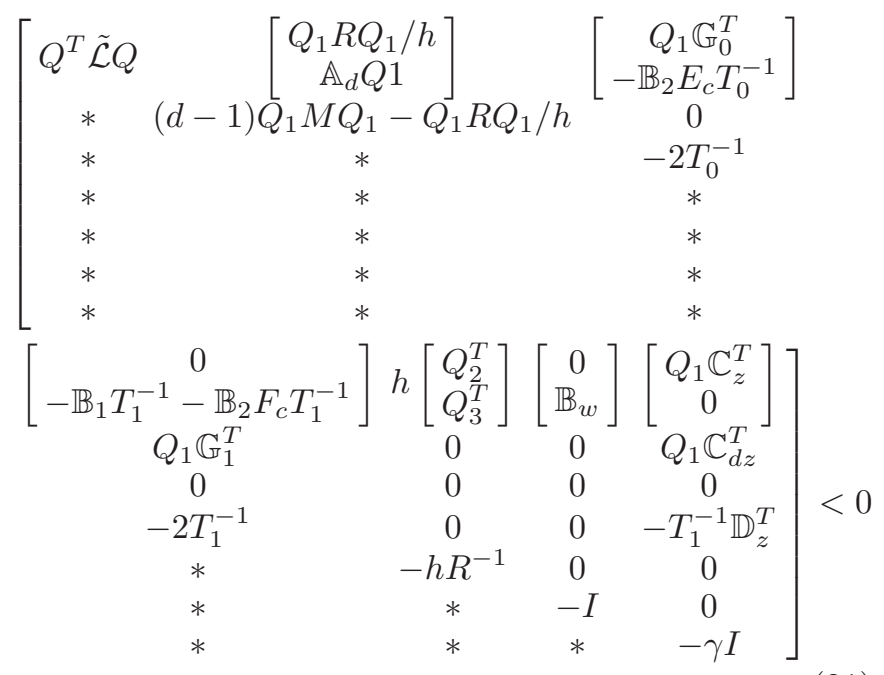

Consider the change of variables: $X=Q_{1} M Q_{1}, U=$ $Q_{1} R Q_{1}, S_{0}=T_{0}^{-1}, S_{1}=T_{1}^{-1}, W_{0}=\mathbb{G}_{0} Q_{1}, W_{1}=$ $\mathbb{G}_{1} Q_{1}, Y_{E}=E_{c} T_{0}^{-1}$ and $Y_{F}=F_{c} T_{1}^{-1}$. Noting that the development of $\left(Q_{1}-R^{-1}\right) R\left(Q_{1}-R^{-1}\right) \geq 0$ implies $R^{-1} \geq 2 Q_{1}-U$, it follows that the LMI condition (10) is obtained.

Suppose that $\phi_{\xi}$ is such that (15) is satisfied. Hence it follows that

$$
\bar{\lambda}\left(P_{1}\right)\left\|\phi_{\xi}\right\|_{c}^{2} \leq \mu^{-1} \Leftrightarrow \max _{\theta \in[-h, 0]} \bar{\lambda}\left(P_{1}\right) \phi_{\xi}(\theta)^{T} \phi_{\xi}(\theta) \leq \mu^{-1} .
$$

On the other hand, $\forall \theta \in[-h, 0]$ one has 


$$
\begin{aligned}
\xi(\theta)^{T} P_{1} \xi(\theta) & \leq \bar{\lambda}\left(P_{1}\right) \xi(\theta)^{T} \xi(\theta) \\
& \leq \max _{\theta \in[-h, 0]} \bar{\lambda}\left(P_{1}\right) \phi_{\xi}(\theta)^{T} \phi_{\xi}(\theta) \leq \mu^{-1}
\end{aligned}
$$

and, if $\phi_{\xi}$ verifies (15), we can conclude that $\xi(\theta) \in$ $\mathcal{E}\left(P_{1}, \mu^{-1}\right), \forall \theta \in[-h, 0]$. Since (11) and (12) ensure that $\mathcal{E}\left(P_{1}, \mu^{-1}\right) \in \mathcal{S}_{0} \cap \mathcal{S}_{1}$, from Lemma 2 it follows that

$$
\Psi(\mathbb{K} \xi(t))^{T} T_{0}\left[\Psi(\mathbb{K} \xi(t))-\mathbb{G}_{0} \xi(t)\right] \leq 0,
$$

$\Psi(\mathbb{K} \xi(t-\tau(t)))^{T} T_{1}\left[\Psi(\mathbb{K} \xi(t-\tau(t)))-\mathbb{G}_{1} \xi(t-\tau(t))\right] \leq 0$ for $t=0$. Then, if (10) is verified, it follows that $J(0)<0$ wich implies $\xi(\Delta t) \in \mathcal{E}\left(P_{1}, \mu^{-1}\right), \Delta t \rightarrow 0$. Following this reasoning, we can conclude that the relations above are indeed verified $\forall t \geq 0$ and hence (10) and (13) ensures that $J(t)<0$ and $\xi(t) \in \mathcal{E}\left(P_{1}, \mu^{-1}\right), \forall t \in[-h, \infty]$, provided that $\phi_{\xi}$ satisfies (15) and $\|w(t)\|_{2}^{2}$ satisfies (2), with $\mu^{-1} \leq \beta+\delta^{-1}$. This concludes the proof of the first and the second items of the Theorem 3 .

Consider now $w(t)=0$. Then, $J(t)<0$ implies that $\dot{V}(t)<-\frac{1}{\gamma} z(t)^{T} z(t)<0$ provided that $\xi(t) \in \mathcal{S}_{0}$ and $\xi(t-\tau(t)) \in \mathcal{S}_{1}$. Hence, from (15) if $\phi_{\xi}$ is such that $\beta<\mu^{-1}$, we have

$$
\xi(t)^{T} P_{1} \xi(t) \leq V(t) \leq V(0) \leq \beta \leq \mu^{-1}
$$

which means that we get $\xi(t) \in \mathcal{E}\left(P_{1}, \mu^{-1}\right)$, for all $t \geq 0$. Because LMIs (11) and (12) are satisfied, it follows $\xi(\bar{t}) \in$ $\mathcal{S}_{0}$ and $\xi(t-\tau(t)) \in \mathcal{S}_{1}$, for all $t \geq 0$. Thus, for any initial condition such that $\beta \leq \mu^{-1}$, it follows effectively that $\dot{V}(t)<0$, which concludes the proof of the third item of Theorem 3 .

Theorem 3 considers the local (or regional) stabilization, in the sense that the computed gains $E_{c}$ and $F_{c}$ ensure asymptotic stability just for the initial conditions satisfying (14) and any disturbance satisfying (2). Provided the open-loop system is asymptotically stable, it can be possible to compute globally stabilizing gains, i.e. the input-to-state stability is ensured for any $w(t) \in \mathcal{L}_{2}$ and the origin of the system is globally asymptotically stable. The next result, which can be seen as a particularization of Theorem 3, allows to address this problem.

Corollary 4. If there exist positive definite matrices $Q_{1}$, $X, U$, positive definite diagonal matrices $S_{0}, S_{1}$, matrices $Q_{2}, Q_{3}, Y_{E}, Y_{F}$ of appropriate dimensions and a positive scalar $\gamma$ satisfying (10) with $W_{0}=W_{1}=\mathbb{K} Q_{1}$, then then there exist anti-windup gains $E_{c}=Y_{E} S_{0}^{-1}$ and $F_{c}=Y_{F} S_{1}^{-1}$ which ensures that:

(1) the trajectories of system (6) are bounded for every initial condition $\phi_{\xi} \in \mathcal{C}_{h}^{\nu}$ and $w(t) \in \mathcal{L}_{2}$;

(2) $\|y\|_{2}^{2} \leq \gamma\|w\|_{2}^{2}+\gamma V(0)$;

(3) when $w=0$, the closed-loop system origin is globally asymptotically stable.

Proof. The proof mimics the one of Theorem 3. In this case, if $\mathbb{G}_{0}=\mathbb{G}_{1}=\mathbb{K}$ it follows that the modified sector condition (9) is verified $\forall \xi(t) \in \mathbb{R}^{n+n c}$ and the global asymptotic stability follows.

Remark 5. In the case where the delay is not mensurable at each instant, it suffices to consider $F_{c}=0$ (which corresponds to fix $Y_{F}=0$ in LMI (10)) in Theorem 3 to ensure the $\mathcal{L}_{2}$-stabilization and/or the internal stabilization of the closed loop systems (6).

\section{OPTIMIZATION PROBLEMS}

In this section we show how the proposed theoretical conditions can be casted into LMI-based optimization problems to determine the anti-windup gains $E_{c}$ and $F_{c}$. In particular, for given bounds $h$ and $d$ on $\tau(t)$ and $\dot{\tau}(t)$, respectively, the following three criteria are considered:

- Maximization of disturbance tolerance: The idea is to maximize the $\mathcal{L}_{2}$ norm bound on the disturbance for which it can be ensured that the system trajectories remain bounded. Considering that the initial condition is null (i.e., $\phi_{\xi}=0, \forall \theta \in[-h, 0]$ ), this can be accomplished by the following convex optimization problem:

$$
\begin{gathered}
\min \mu \\
\text { subject to } \\
(10) \text { to }(12) \text {. }
\end{gathered}
$$

- Maximization of disturbance attenuation: For a nonnull bound on the $\mathcal{L}_{2}$ norm of the admissible disturbances (given by $\mu^{-1}=\delta^{-1}$ ), the idea is to minimize the upper bound for the $\mathcal{L}_{2}$ gain of $w(t)$ on $z(t)$. Considering that the initial condition is null, this can be obtained from the solution of:

$$
\begin{gathered}
\min \gamma \\
\text { subject to } \\
(10) \text { to }(13) .
\end{gathered}
$$

- Maximization of the set of admissible initial conditions: We consider here the disturbance free case, i.e. $w(t)=0$. In order to ensure the stability of system (1) by using the Theorem 3, the admissible initial conditions must verify condition (14) with $\beta \leq \mu^{-1}$.

Consider $\|\phi(\theta)\|_{c}^{2}=\kappa_{1}$ and $\|\dot{\phi}(\theta)\|_{c}^{2}=\kappa_{2}$ and note that as the maximal eigenvalues of $Q_{1}^{-1}$, $Q_{1}^{-1} U Q_{1}^{-1}$ and $Q_{1}^{-1} X Q_{1}^{-1}$ decreases, the value of $\kappa_{1}$ and $\kappa_{2}$ for which (14) is verified increases. Hence, the problem of finding $E_{c}$ and $F_{c}$ leading to the maximization of the region of stability of the closed-loop system can be achieved by minimizing these maximal eigenvalues. Consider

$$
\begin{gathered}
{\left[\begin{array}{cc}
\lambda_{Q_{1}} I & I \\
* & Q_{1}
\end{array}\right] \geq 0, \quad\left[\begin{array}{cc}
\lambda_{X} I & I \\
* & 2 Q_{1}-X
\end{array}\right] \geq 0} \\
{\left[\begin{array}{cc}
\lambda_{U} I & I \\
* & 2 Q_{1}-U
\end{array}\right] \geq 0}
\end{gathered}
$$

From the fact that $R^{-1} \geq 2 Q_{1}-U$ and $M^{-1} \geq$ $2 Q_{1}-X$ it follows that these LMIs are respectively equivalent to $\lambda_{Q_{1}} \geq \bar{\lambda}\left(Q_{1}^{-1}\right), \lambda_{X} \geq \bar{\lambda}\left(Q_{1}^{-1} X Q_{1}^{-1}\right)$ and $\lambda_{U} \geq \bar{\lambda}\left(Q_{1}^{-1} U Q_{1}^{-1}\right)$.

Hence, the following optimization problem can be considered:

$$
\begin{gathered}
\min \beta_{1} \lambda_{Q 1}+\beta_{2} \lambda_{X}+\beta_{3} \lambda_{U} \\
\text { subject to } \\
(10),(11),(12) \text { and }(24)
\end{gathered}
$$

where $\beta_{1}, \beta_{2}$ and $\beta_{3}$ are weights that should be tuned in order to satisfy some trade-off between $\kappa_{1}$ and $\kappa_{2}$.

\section{NUMERICAL EXAMPLE}

Consider system (1) given by the following matrices: $A=0.1, B=B_{w}=1, C_{y}=C_{z}=-5, D_{z}=-20, u_{0}=1$ 
and a stabilizing PI controller described by:

$$
A_{c}=0, B_{c}=-0.2, C_{c}=1, D_{c}=-2
$$

Since the open loop system is unstable, we apply the local stability results proposed in Theorem 3 .

In Table 1 is presented the result from the solution of Problem (22) with $d=0.1$ and different values of $h$ considering two situations: the value of $\tau(t)$ is known at each time and can be used to calculate $\Psi(\mathbb{K} \xi(t-\tau(t)))$ (in this case $F_{c} \neq 0$ ); the value of $\tau(t)$ is unknown at each time, i.e. it is not possible to compute $\Psi(\mathbb{K} \xi(t-\tau(t))$ ) (in this case $\left.F_{c}=0\right)$. It is easy to see that the solution with $F_{c} \neq 0$ is less conservative since the minimal value of $\mu$ to which is possible to solve Problem (22) is smaller than the one obtained for $F_{c}=0$.

\begin{tabular}{c|c|c}
$h$ & $\mu @ F_{c} \neq 0$ & $\mu @ F_{c}=0$ \\
\hline \hline 0.0100 & $8.3942 \times 10^{-13}$ & $5.2113 \times 10^{-11}$ \\
0.0500 & 0.0845 & 0.1978 \\
0.1000 & 0.1375 & 0.2609 \\
0.5000 & 0.1986 & 0.9686 \\
0.5995 & $0.8906 \times 10^{3}$ & $3.2892 \times 10^{3}$ \\
\hline
\end{tabular}

Table 1. Results for Problem (22)

In Table 2 is presented the result from the solution of Problem (23) with $h=0.1$ and $d=0.1$ for different values of $\frac{1}{\delta}=\frac{1}{\mu}$. Also in this case, less conservative results for $\gamma$ are obtained with $F_{c} \neq 0$ since the minimal value of $\mu$ (which implies in larger disturbance bounds) to which is possible to solve Problem (23) is smaller than the one obtained for $F_{c}=0$.

\begin{tabular}{c|c|c}
$\mu$ & $\gamma @ F_{c} \neq 0$ & $\gamma @ F_{c}=0$ \\
\hline \hline 0.1371 & $9.3049 \times 10^{5}$ & - \\
0.2607 & 386.2742 & $7.6351 \times 10^{5}$ \\
1 & 386.1440 & 430.1776 \\
10 & 386.1427 & 386.4972 \\
100 & 386.0567 & 386.3698 \\
1000 & 386.0510 & 386.3686 \\
\hline \hline
\end{tabular}

$\overline{\text { Table 2. Results for Problem (23) }}$

\section{CONCLUSIONS}

The problem of anti-windup design for linear systems with time-varying input delay and actuator saturation has been addressed. An anti-windup structure with two static compensators has been considered. Based on a LyapunovKrasovskii functional and a modified sector approach, global and local stabilization conditions directly in the LMI form were derived to ensure both the external and the internal stability of the closed-loop system in the presence of $\mathcal{L}_{2}$-bounded disturbances. Provided that the maximal value of the time-varying delay and its derivative are known, convex optimization problems were proposed to compute the anti-windup gains aiming at three different control objectives. Simulation examples are provided to illustrate the effectiveness of the proposed solution.

The extension of the results to consider systems with both input and output time-varying delays are in study. This case is particularly interesting in networked control applications.

\section{REFERENCES}

Fridman, E. and Shaked, U. (2002). An improved stabilization method for linear time-delay systems. IEEE Transactions on Automatic Control, 47(11), 1931-1937.

Fridman, E. and Shaked, U. (2003). Delay-dependent stability and $\mathcal{H}_{\infty}$ control: constant and time-varying delays. International Journal of Control, 76(1), 48-60.

Gomes da Silva Jr., J.M., Seuret, A., Fridman, E., and Richard, J.P. (2010). Stabilisation of neutral systems with saturating control inputs. International Journal of Systems Science, 1-11.

Gomes da Silva Jr., J.M., Tarbouriech, S., and Garcia, G. (2006). Anti-windup design for time-delay systems subject to input saturation. an lmi-based approach. European Journal of Control, 12(6), 622-634.

Gu, K., Kharitonov, V., and Chen, J. (2003). Stability of time-delay systems. Birkhäuser.

Mahmoud, M.S. (2009). Improved robust stability and feedback stabilization criteria for time-delay systems. IMA Journal of Mathematical Control and Information, 26(4), 451-466.

Niculescu, S.J., Fu, M., and Li, H. (1997). Delaydependent closed-loop stability of linear systems with input delay: an lmi approach. In Proceedings of the 36th IEEE Conference on Decision and Control ( $\left.\mathrm{CDC}^{7} 7\right)$, volume 2, $1623-1628$ vol.2.

Park, J.K., Choi, C.H., and Choo, H. (2000). Dynamic anti-windup method for a class of time-delay control systems with input saturation. International Journal of Robust and Nonlinear Control, 10(6), 457-488.

Park, J.K., Lim, H., Basar, T., and Choi, C.H. (2003). Anti-windup compensator for active queue management in tcp networks. Control Engineering Practice, 11(10), $1127-1142$.

Richard, J.P. (2003). Time-delay systems: an overview of some recent advances and open problems. Automatica, 39, 1667-1604.

Shaked, U., Yaesh, I., and de Souza, C.E. (1998). Bounded real criteria for linear time-delay systems. IEEE Transactions on Automatic Control, 43(7), 1016 -1022.

Tarbouriech, S., Gomes da Silva Jr., J.M., and Garcia, G. (2003). Delay-dependent anti-windup loops for enlarging the stability region of time-delay systems with saturating inputs. Trans. ASME - J. of Dyn. Syst., Meas.and Contr., 125(1), 265-267.

Tarbouriech, S., Gomes da Silva Jr., J.M., and Garcia, G. (2004). Delay-dependent anti-windup strategy for linear systems with saturating inputs and delayed outputs. International Journal of Robust and Nonlinear Control, $14,665-682$.

Tarbouriech, S. and Turner, M. (2009). Anti-windup design: An overview of some recent advances and open problems. IET Control Theory and Applications, 3(1), $1-19$.

Zaccarian, L., Nesic, D., and Teel, A.R. (2005). $\mathcal{L}_{2}$ antiwindup for linear dead-time systems. Systems \& Control Letters, 54(12), 1205 - 1217.

Zhang, X.M., Wu, M., She, J.H., and He, Y. (2005). Delaydependent stabilization of linear systems with timevarying state and input delays. Automatica, 41(8), 14051412. 